\title{
Feedback Control of an Active Sound Insulation Unit*
}

\author{
Naoki KANAMORI**, Masaharu NISHIMURA** and Takumi MISHIRO***
}

\begin{abstract}
This paper describes designs of structures and control systems of an active sound insulation unit consisting of active cells which are individually controlled as a single input single output system. Two types of Active Sound Insulation Cell (ASIC) using lightweight card-type speakers were manufactured for trial. It became clear that ASIC can reduce the transmitted noise whose sound field inside the cell is uniform. That means that the size of ASIC should be less than quarter wavelength of controlled sound and secondary noise sources should be properly arranged. The feedback control system for ANC was systematically designed by $\mathrm{H}-$ infinity control theory, and the sound attenuating performance was verified by the simulation.
\end{abstract}

Key Words: Measurement and Control, Active Wave Control, Acoustic, Active Noise Control, Feedback Control, Sound Insulation, H-Infinity Control

\section{Introduction}

Active noise control of large space needs a multiple channel control system. And it is supposed to be too difficult to be applied to the real space because of its complexity and expensive cost. Then it is considered to be more realistic to control acoustic boundary by setting simple and individually controlled acoustic units side by side at the boundary. For example, Active Acoustic Treatment (AAT) was proposed for absorbing sound at walls and Active Soft Edge (ASE) was developed for attenuating sound refraction at noise barriers by the authors ${ }^{(1)-(3)}$.

On the other hand, light and high performance sound insulation wall is needed especially for vehicle body. And some active noise control system is expected to be effective for increasing sound transmission loss of panel at low frequency region without adding mass. Several researches were already reported, and effective results were obtained $^{(4)-(7)}$. But these were not aimed to be light and simple. Recently, researches of the active insulation system using the lightweight actuator are also advanced ${ }^{(8)}$.

The purpose of this research is to develop distributed control system which consists of many individually controlled light units for increasing sound insulation of panel.

* Received 30th January, 2006 (No. 05-4286)

** Department of Mechanical Engineering, Tottori University, 4-101 Koyama-minami, Tottori-city, Tottori 6808552, Japan. E-mail: kanamori@mech.tottori-u.ac.jp; mnishimura@mech.tottori-u.ac.jp

*** Mobile Energy Company, Sanyo Electric Co., Ltd., 55 Keihan-Hondori 2-Chome, Moriguchi-city, Osaka 5708677, Japan. E-mail: misiro@sm.energy.sanyo.co.jp
In this paper, two types of unit which is called Active Sound Insulation Cell (ASIC) were proposed and manufactured for trial. They used light card-type speakers. And their performances of sound insulation were evaluated by simple experiments using a reverberation chamber. It became clear that ASIC can reduce the transmitted noise whose sound field inside the cell is uniform. That means the size of ASIC should be less than quarter wavelength of controlled sound. The sound attenuation control experiment using Filtered-x-LMS algorithm was conducted to ASIC, and the about $10 \mathrm{~dB}$ sound attenuation effect was acquired by the frequency band $400 \mathrm{~Hz}$ or less.

However, a reference signal cannot be acquired when a cell is actually used. The controller needed to be made into the feedback system only using an error signal. The feedback controller for ANC was systematically designed by $\mathrm{H}$-infinity control theory, and the stable sound attenuating performance of about $4 \mathrm{~dB}$ of maximum was obtained below $500 \mathrm{~Hz}$ by the simulation.

\section{Basic Concept of Active Sound Insulation Unit}

Schematic drawing of the typical examples of active sound insulation systems using ASIC is shown in Fig. 1. These examples have double walls whose inner space is divided to many cells. Uniform sound field exists in each cell below the target frequency, and sound pressure level is actively controlled to zero. There exist light card-type speakers, an error microphone and a controller inside the cell. Speakers are controlled to minimize the output of the error microphone, according to incident sound. Each cell is simply controlled individually as a single input single output system. Feedback control is generally adopted 
because the information of incident sound cannot be obtained. Technical issues to realize these kinds of ASIC are as follows:

(1) Make clear the relation between controllable frequency and cell size.

(2) Optimize the position of speakers and an error microphone.

(3) Optimize the controller.

(4) Reduce cross-talk between neighboring cells.

(5) Reduce solid-borne noise.

(6) Optimize the material of cells.

In this paper, the first three items were studied.

\section{Manufactured ASIC and Test Procedure}

\subsection{Outlines of manufactured ASIC}

Two types of ASIC were manufactured and tested for trial. Both types of ASIC used card-type speakers because of reducing weight. One is Type-A in which card-type speakers are set along a side wall parallel to the sound path as shown in Fig. 1. The other is Type-B in which card-type

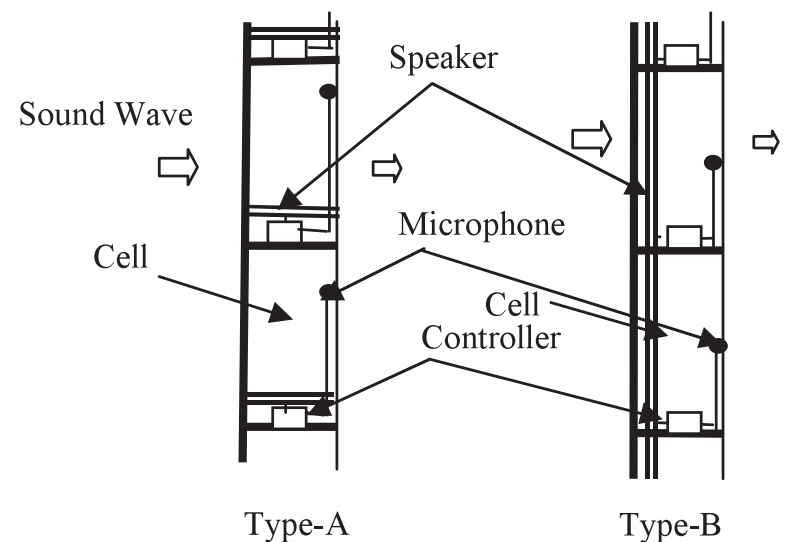

Fig. 1 Two types of active sound insulation unit speakers are set perpendicular to the sound path as shown in Fig. 1.

The size of a manufactured ASIC is $240 \mathrm{~mm}$ square and the air-space between double walls is $70 \mathrm{~mm}$. The flames are made of wood whose thickness is $5 \mathrm{~mm}$. Double walls are made of aluminum plate whose thickness is $0.5 \mathrm{~mm}$. Light card-type speakers (Panasonic WM-R57A) are used as the secondary source. Type-A uses 4 speakers set with $15 \mathrm{~mm}$ back space and actuated by same signal, which is shown in Fig. 2. And 9 minute microphones are set in the cell to measure the sound field in the cell as shown in Fig. 2. One of them is used as an error microphone. In Type-B, 16 speakers are set and actuated by same signal as shown in Fig. 3.

\subsection{Test apparatus}

Test apparatus is shown in Fig. 4. In order to realize random sound incident condition, a reverberation chamber was constructed which was $800 \mathrm{~mm}$ wide and $1135 \mathrm{~mm}$ high. A manufactured ASIC was attached to the chamber window. A speaker set in the chamber was used as a primary source. And a set of card-type speakers in the ASIC was actuated as a secondary source. Monitoring microphones were set at $50 \mathrm{~mm}$ and $500 \mathrm{~mm}$ apart from the $\operatorname{ASIC}\left(\mathrm{m}_{1}\right.$ and $\left.\mathrm{m}_{2}\right)$ to evaluate the sound attenuating performance.

\subsection{Acoustic characteristics of active cell}

Based on our concept, ASIC should be controlled as a single input single output system. That means that the sound field inside an ASIC should be uniform (same gain and same phase). Then transfer functions between every two microphones' outputs inside the cell were measured in both cases of the primary source excitation and the secondary source excitation.

3. 4 Prediction of sound attenuating performance

Sound attenuating performance of ASIC was predicted at the monitoring positions $\mathrm{m}_{1}$ and $\mathrm{m}_{2}$ based on

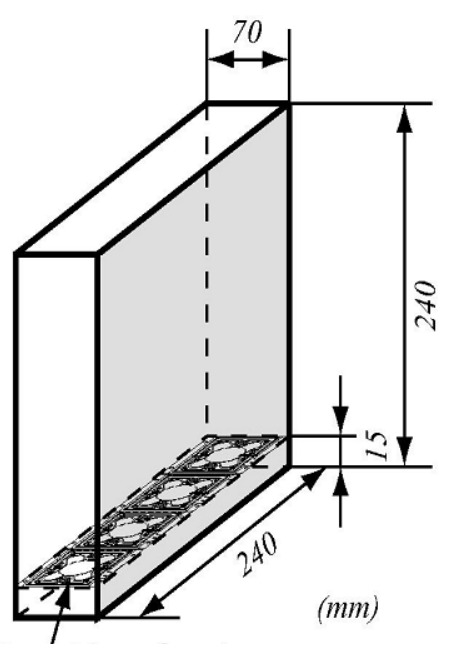

Card type speakers
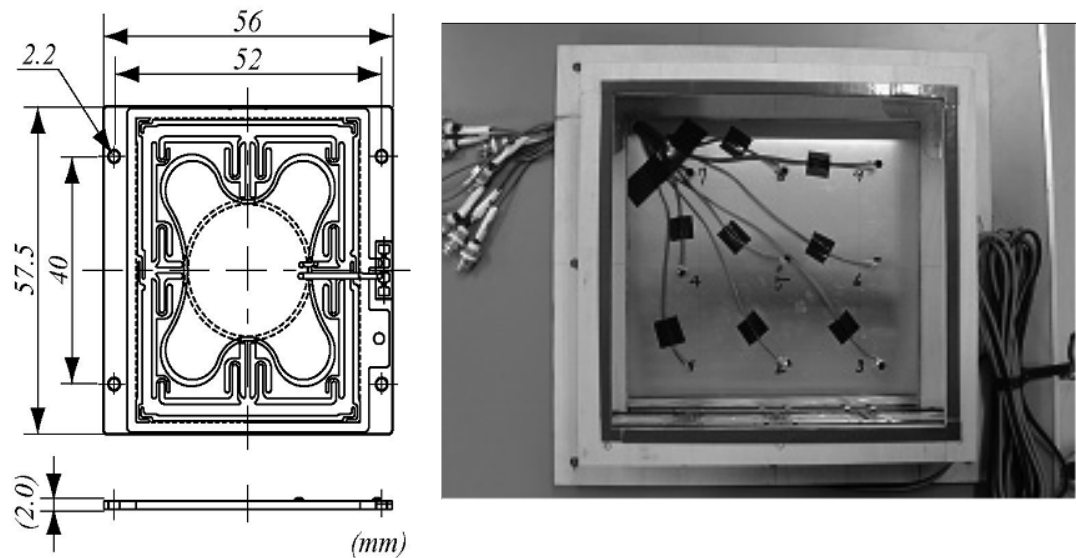

Fig. 2 Active sound insulation cell (Type-A) 

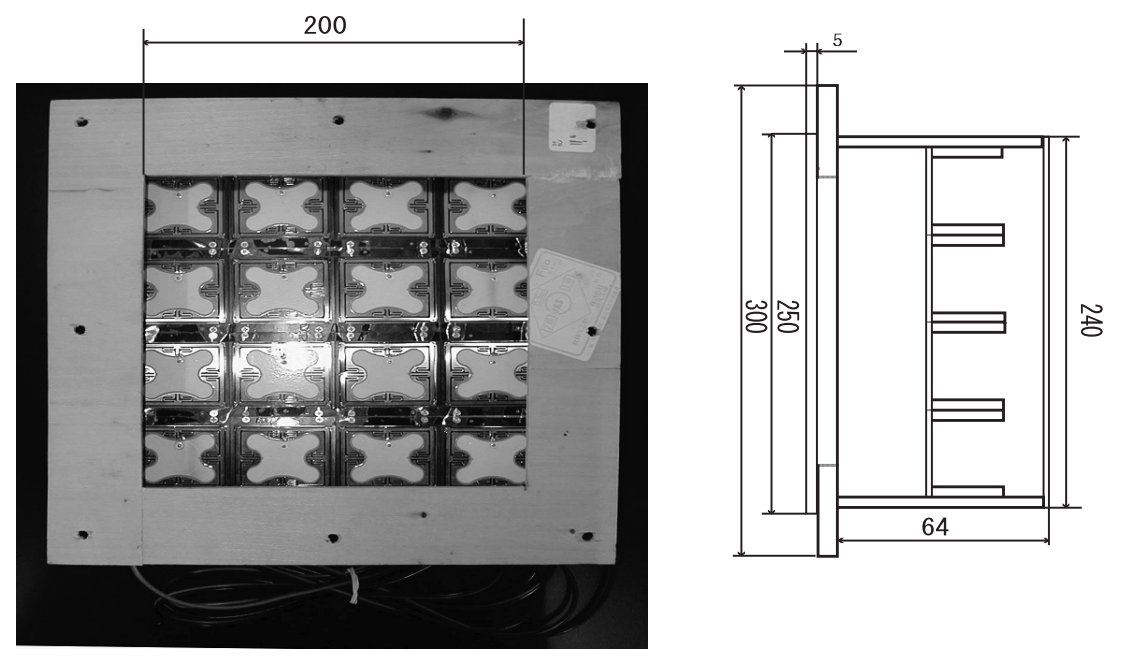

Fig. 3 Active sound insulation cell (Type-B)

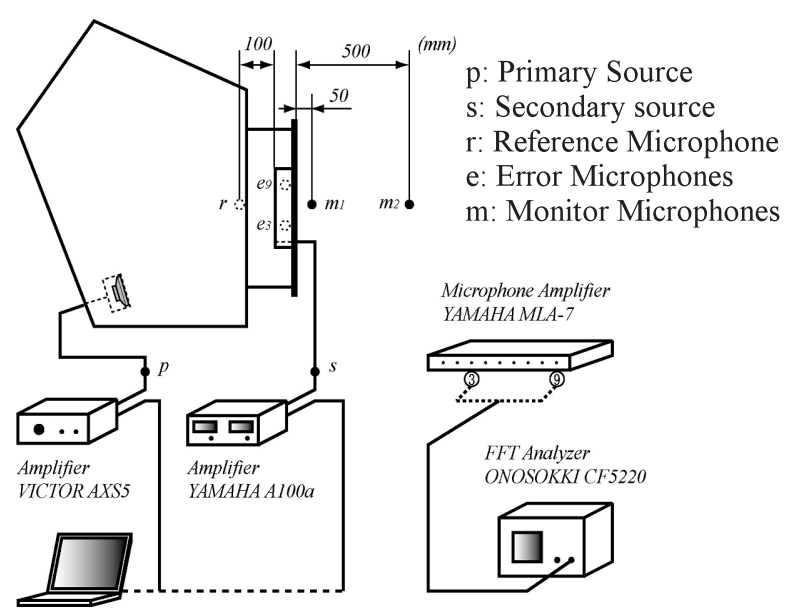

Fig. 4 Test apparatus

the following method by assuming that an error signal became zero. The output of the error microphone $E(\omega)$ is explained as follows:

$$
E(\omega)=H p e_{k}(j \omega) U(\omega)+H s e_{k}(j \omega) V(\omega)
$$

Where $U(\omega)$ is input signal to the primary source and $V(\omega)$ is input signal to the secondary source. $H p e_{k}$ and $H s e_{k}$ are respectively transfer function from the primary source to an error microphone and that from the secondary source to the error microphone. Therefore, $E(\omega)=0$ needs the following relationship:

$$
V(\omega)=\frac{\operatorname{Hpe}_{k}(j \omega)}{H s e_{k}(j \omega)} U(\omega)
$$

On the other hand, sound pressure $M(\omega)$ at a monitoring position in the case of active control is obtained as follows:

$$
M(\omega)=H_{p m}(j \omega) U(\omega)+H s m_{i}(j \omega) V(\omega)
$$

Where $\mathrm{Hpm}_{i}$ and $\mathrm{Hsm}_{i}$ are respectively transfer function from the primary source to a monitor microphone and that from the secondary source to the monitor microphone. If the relation of Eq. (2) is realized, Eq. (3) becomes as follows:

$$
\begin{aligned}
& M(\omega)=\left(H_{i p m_{i}}(j \omega)-H s m_{i}(j \omega) \frac{H p e_{k}(j \omega)}{H s e_{k}(j \omega)}\right) U(\omega) \\
& =H p e_{k} m_{i}(j \omega) U(\omega)
\end{aligned}
$$

In the case of non-control, sound pressure at the monitoring position $M_{0}(\omega)$ is explained below:

$$
M_{0}(\omega)=H_{p}(j \omega) U(\omega)
$$

Then sound attenuation Attpe $_{k} m_{i}$ is obtained as follows:

$$
\begin{aligned}
& \text { Attpe }_{k} m_{i}=-10 \log _{10} \frac{S_{M M}}{S_{M_{0} M_{0}}} \\
& =-10 \log _{10} \frac{M^{*}(\omega) M(\omega)}{M_{0}^{*}(\omega) M_{0}(\omega)} \\
& =-10 \log _{10} \frac{H^{*} p e_{k} m_{i}(j \omega) H p e_{k} m_{i}(j \omega)}{H^{*} p m_{i}(j \omega) H p m_{i}(j \omega)}
\end{aligned}
$$

According to these equations, we can evaluate the performance of ASIC by measured transfer functions.

\section{Test Results of Type-A}

\section{1 Sound field in the cell}

Figure 5 shows transfer functions between microphones' outputs in the cell in the case of primary source excitation. It is found that roughly uniform sound field exists below $400 \mathrm{~Hz}$ except some special frequencies. 'Below $400 \mathrm{~Hz}$ ' means the frequency region whose wavelength is more than 4 times of the cell dimension. Figure 6 shows those in the case of secondary source excitation. In this case, phase of each microphone is also roughly uniform below $400 \mathrm{~Hz}$. But gain is uniform only around $200 \mathrm{~Hz}$ and $370 \mathrm{~Hz}$. Where the resonant frequency input of the double walls (masses) connected by air space (spring) is $200 \mathrm{~Hz}$. And $370 \mathrm{~Hz}$ is considered to be the secondary resonance. The discrepancy of gain at other frequencies is supposed to be caused by biased arrangement of card-type speakers. And the discrepancy below $100 \mathrm{~Hz}$ is because of insufficient sound radiating performance of card-type speakers. 


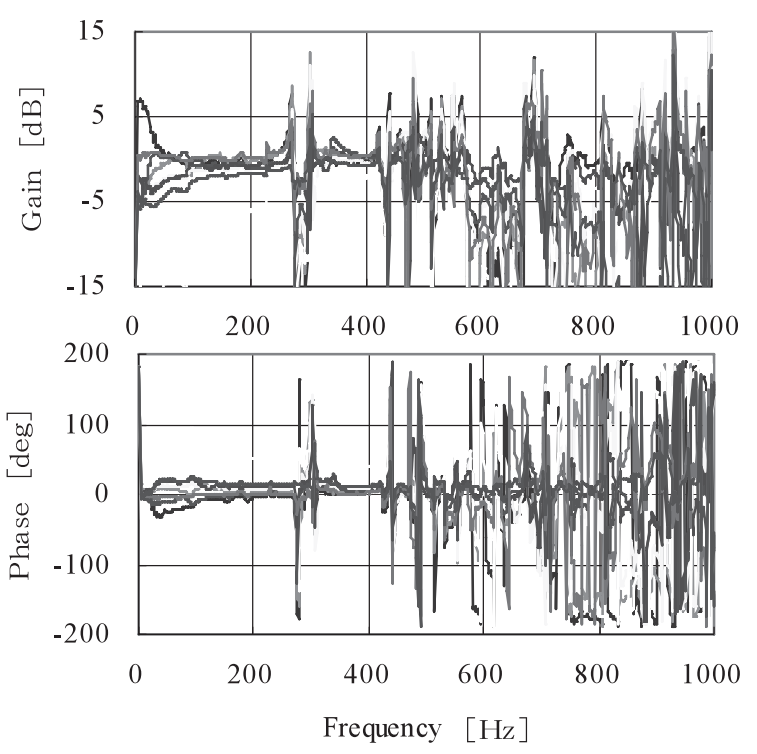

Fig. 5 Transfer functions from $\mathrm{e}_{9}$ to $\mathrm{e}_{i}$ (at primary source excitation: Type-A)
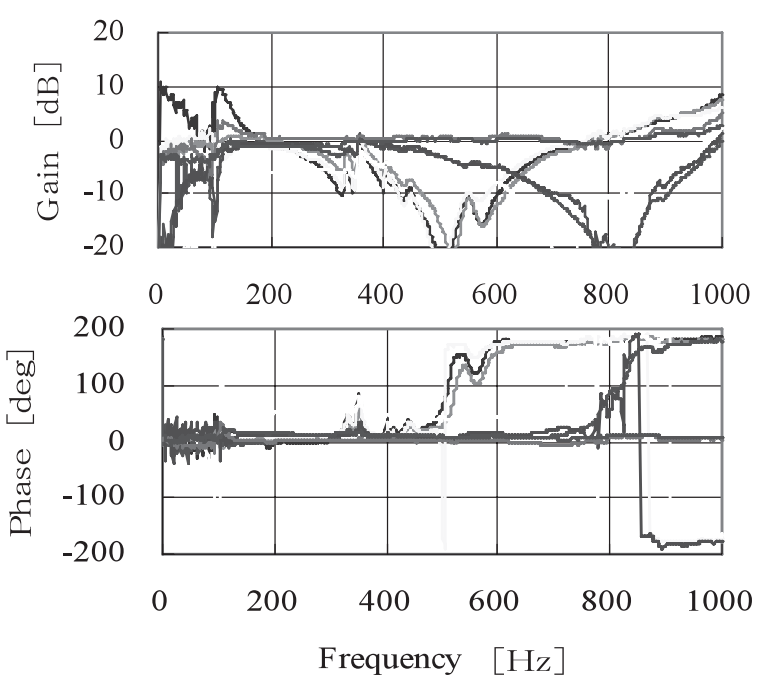

Fig. 6 Transfer functions from $e_{9}$ to $e_{i}$ (at secondary source excitation: Type-A)

\subsection{Sound attenuating performance prediction}

Sound attenuating performance was predicted according to the above method. Sound power spectra at $\mathrm{m}_{1}$ with and without control are shown in Fig. 7. Peak spectra from $170 \mathrm{~Hz}$ to $200 \mathrm{~Hz}$ and around $370 \mathrm{~Hz}$ are attenuated by the active control. But other spectra cannot be reduced. The result clearly shows that the sound attenuating performance was acquired in the frequency band whose inside of the cell is uniform sound field. It was confirmed to be necessary to generate uniform sound field inside the cell by the secondary source.

\section{Test Results of Type-B}

\subsection{Sound field in the cell}

In order to study how we can realize uniform sound field inside the cell by the secondary source, Type-B ASIC

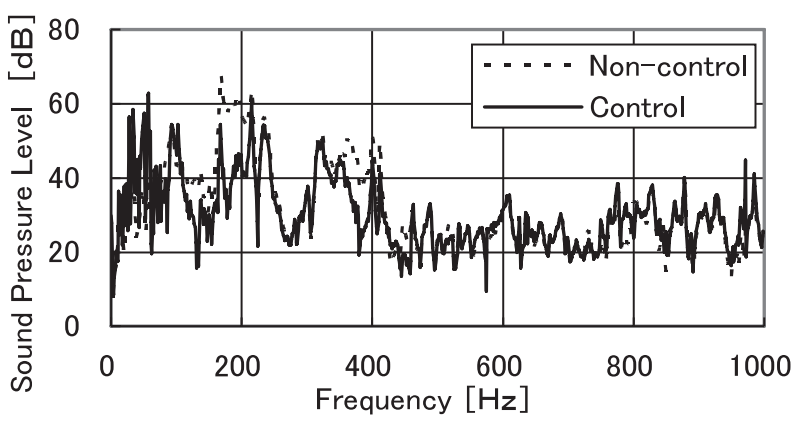

Fig. 7 Sound attenuating performance of the active control (simulation: Type-A)
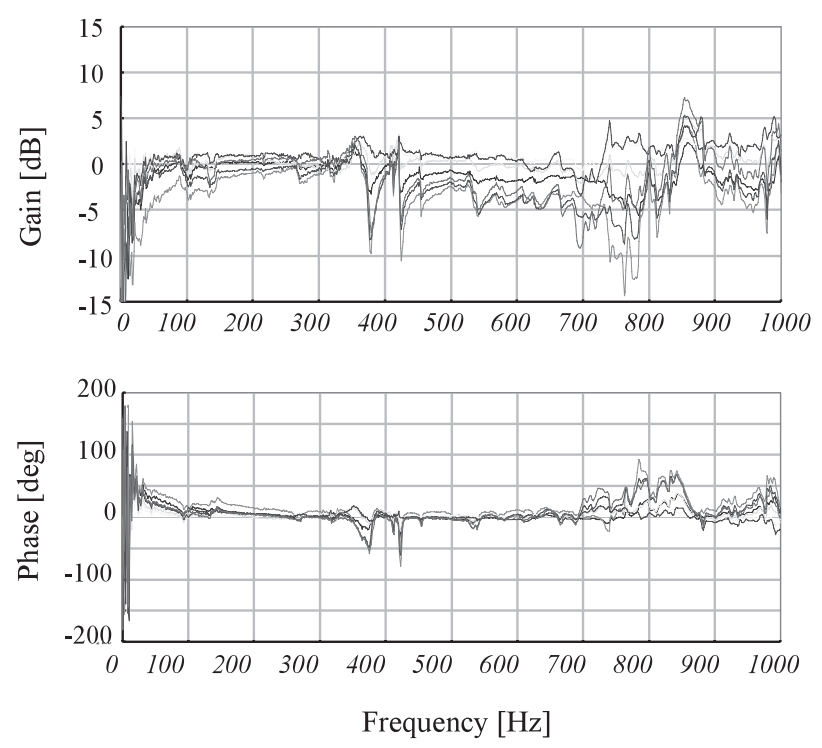

Fig. 8 Transfer functions from $\mathrm{e}_{1}$ to $\mathrm{e}_{i}$ (at secondary source excitation: Type-B)

was tried to be manufactured and tested. In this case a speaker plate constructed by 16 card type speakers was set in the cell perpendicularly to the sound path. Figure 8 shows transfer functions between microphones' outputs in the cell when the secondary source is operating.

\subsection{Sound attenuating performance prediction and experiment}

Figure 9 shows the predicted sound attenuating performance of the Type-B ASIC. It is found that sound attenuating frequency region become wider than Type-A.

Then, the sound attenuating control experiments are performed to the cell of Type-B using the Filtered-x-LMS control system. The experimental setup shown in Fig. 10 is controlled by Filtered-x-LMS algorithm which used the noise generator signal $r$ and the error microphone signal $\mathrm{e}_{5}$. The Sampling frequency is $2 \mathrm{kHz}$ and the tap length is 512. Figure 9 shows the experimental results when setting a monitor point to $\mathrm{m}_{1}$. Notice is about $10 \mathrm{~dB}$ sound attenuating performance below $400 \mathrm{~Hz}$, and the control result as prediction. However, the frequency band without sound attenuation exists. As the reason, it is possible that the 


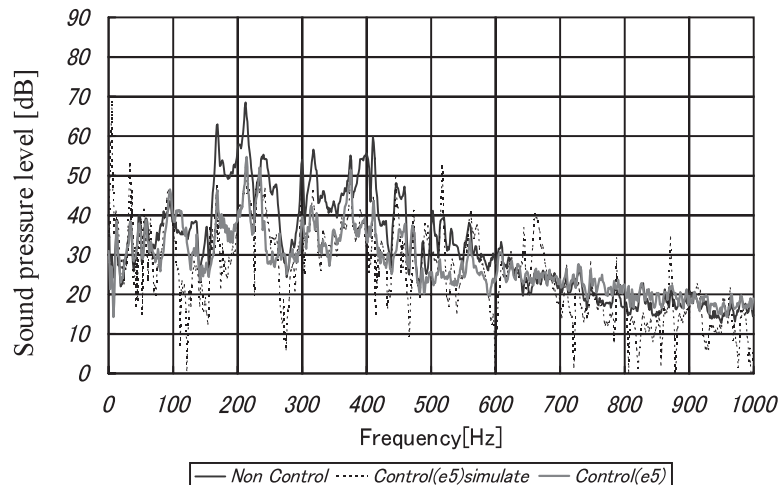

Fig. 9 Sound attenuating performance of the active control (Type-B)

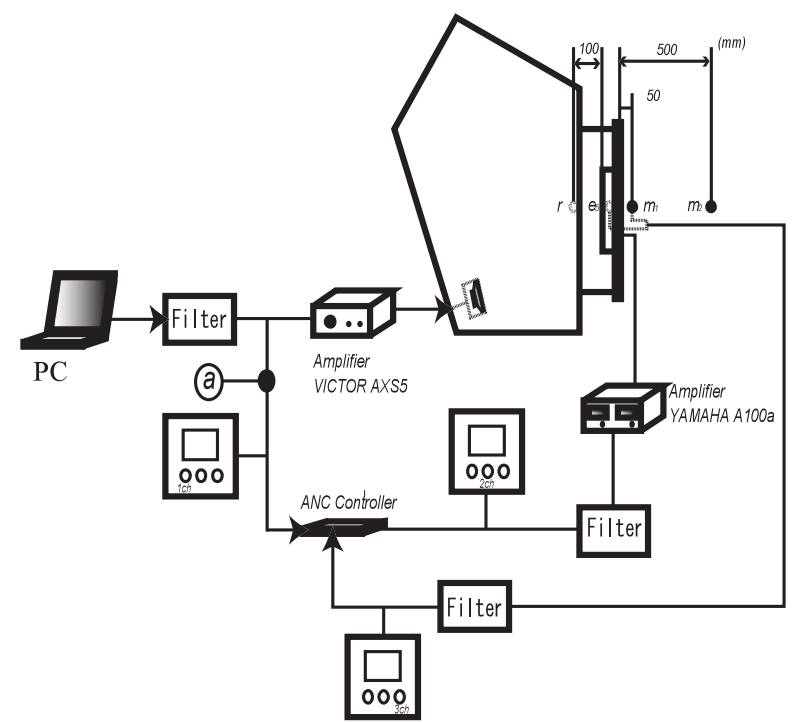

Fig. 10 Experimental setup for ANC

tap length of a FIR filter cannot express the acoustic characteristics of the reverberation chamber. In the following paragraph, Type-B is used.

\section{Feedback Controller Design for ASIC}

\section{1 Structure}

In feedback control, only an error microphone is referred. Figure 11 is the block diagram of the feedback control system. Where $\mathrm{P}$ is a plant and $\mathrm{C}$ is a controller. Signal $\mathrm{d}$ is incident sound and e is an error. Signal $\mathrm{r}$ is desired sound attenuation and is set to 0 . The controller has forced to control the point of the error microphone using the signal detected with the error microphone. Therefore, even when any incidence sound is input, the feedback system must attenuate sound without diverging.

\subsection{Controller design via tandem compensators}

The design of Controller $\mathrm{C}$ was realized by connecting plural phase compensators in series to plant $\mathrm{P}$. Controller $\mathrm{C}$ designed is set to $\mathrm{C}_{1}$. The notes by the design of the open-loop transfer function are as follows:

(1) The gain margin for stability must exist.

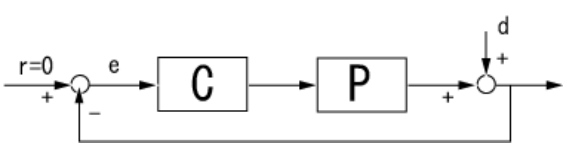

Fig. 11 Block diagram of the feedback control system
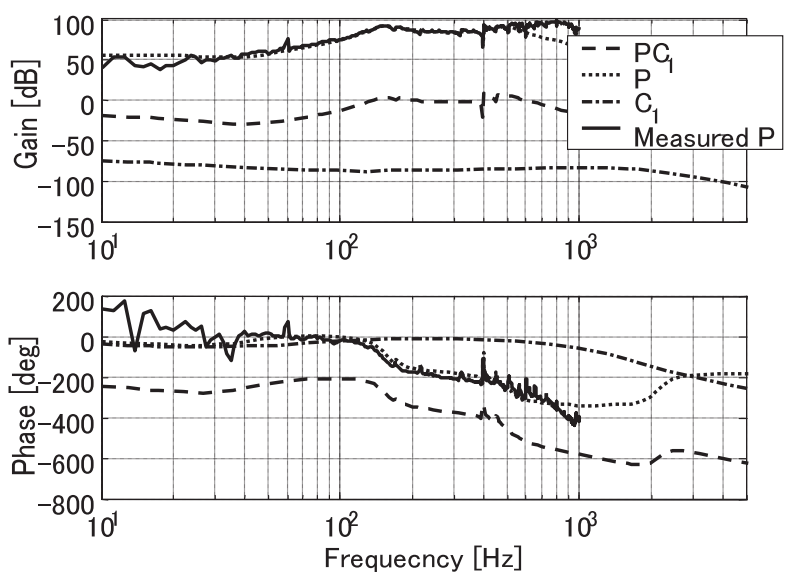

Fig. 12 Plant $\mathrm{P}$, controller $\mathrm{C}_{1}$ and open-loop transfer function $\mathrm{PC}_{1}$

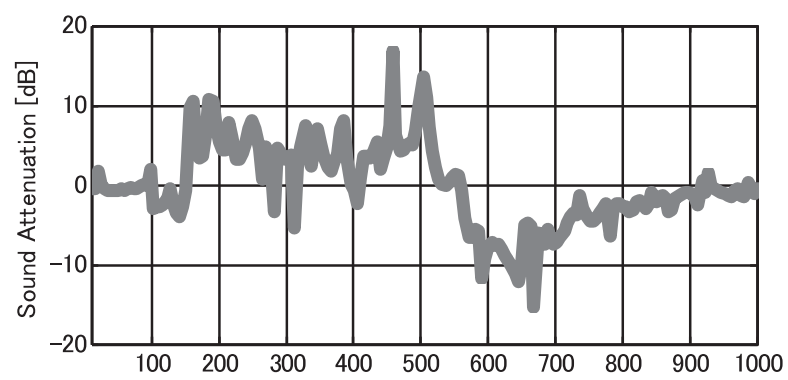

Fig. 13 Simulation results by open-loop method

(2) In the frequency band of sound attenuation, the gain is above $0 \mathrm{~dB}$.

( 3 ) On a high frequency side, fully drop the gain.

Figure 12 is bode diagrams of a plant $\mathrm{P}$, a designed controller $\mathrm{C}_{1}$ and the open-loop transfer function $\mathrm{PC}_{1}$. The result of the sound attenuating simulation using this controller $\mathrm{C}_{1}$ is Fig. 13. The transfer function from the noise signal $\mathrm{d}$ to the error signal $\mathrm{e}$ is shown in that figure. The primary sound source $\mathrm{d}$ was made from the white noise which passed the 100 to $500 \mathrm{~Hz}$ band path filter. Since the gain is above $0 \mathrm{~dB}$ between $150 \mathrm{~Hz}$ and $550 \mathrm{~Hz}$, sound attenuating performance is realizable.

\subsection{Controller design via $\mathrm{H}$-infinity control theory}

The controller $\mathrm{C}_{1}$ is designed for the only plant $\mathrm{P}$. However, the actual plant $\mathrm{P}$ is changed by temperature change or a manufacture error of a cell. Perturbation of the plant may make a closed-loop system unstable, then it may diverge. For practical use, the controller must guarantee stability also to such perturbation of the plant. On the other hand, we want to design a controller from the specification of the desired sound attenuation. Then, we applied H-infinity control theory. 


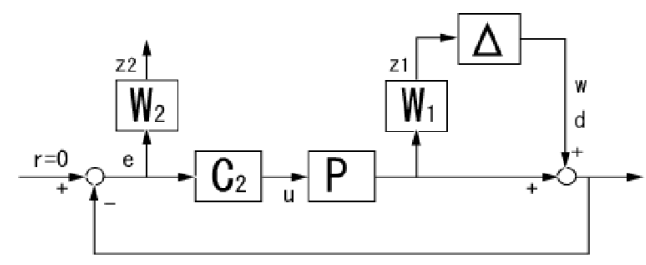

Fig. 14 Block diagram for H-infinity control

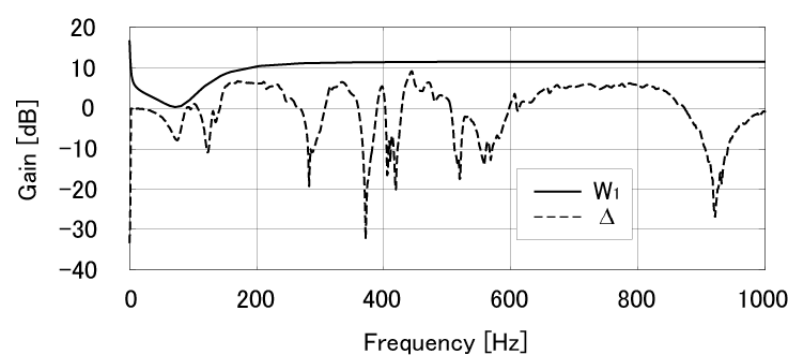

Fig. 15 Multiplicative uncertainty and weighting function $W_{1}$

The specification of a sound attenuation and a stability margin can be given as maximum of $\mathrm{H}$-infinity norms of the closed-loop system. And the controller which fulfills those conditions is systematically found by H-infinity control theory. Figure 14 is the block diagram for $\mathrm{H}$ infinity control. $\mathrm{P}$ is a nominal plant and $\Delta$ is uncertainty expressed with $\|\Delta\|_{\infty} \leq 1$.

Two weighting functions $W_{1}$ and $W_{2}$ are determining the maximum of multiplicative perturbation and specifying sound attenuation respectively. And $\mathrm{C}_{2}$ is a found controller. The closed-loop transfer functions $T$ and $S$ are defined as follows:

$$
\begin{aligned}
& T=\frac{P C_{2}}{1+P C_{2}} \\
& S=\frac{1}{1+P C_{2}}=1-T
\end{aligned}
$$

The design conditions of a controller are guaranteeing robust stability in case a plant has uncertainty, and that the error signal e becomes smaller than the signal d of a primary sound source, and are expressed with inequality (9) and (10), respectively:

$$
\begin{aligned}
& \left\|W_{1} T\right\|_{\infty}<1 \\
& \left\|W_{2} S\right\|_{\infty}<1
\end{aligned}
$$

The weighting functions $W_{1}$ and $W_{2}$ are decided as follows, respectively.

First, the plant containing multiplicative uncertainty is expressed with:

$$
\tilde{P}=\left(1+\Delta W_{1}\right) P
$$

The supremum of the uncertainty is limited by inequality (12).

$$
\left|\frac{\tilde{P}(j \omega)}{P(j \omega)-1}\right|<W_{1}(j \omega), \quad \forall \omega
$$

Deciding $W_{1}$ means deciding the supremum of the closedloop transfer function $T . W_{1}$ is decided as shown in Fig. 15.

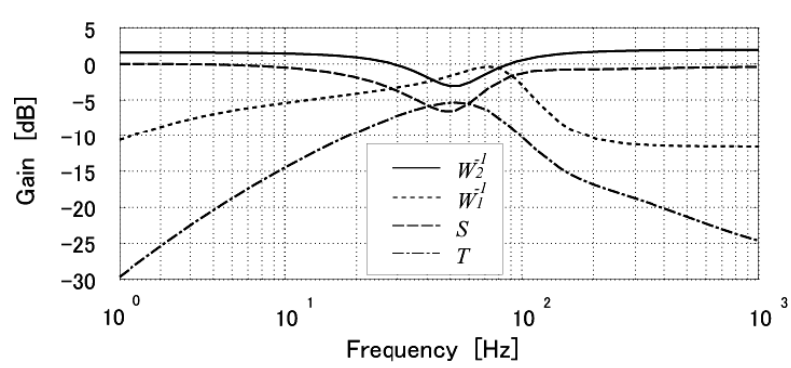

Fig. 16 Weighting functions $W_{1}$ and $W_{2}$ and closed-loop transfer functions $S$ and $T$

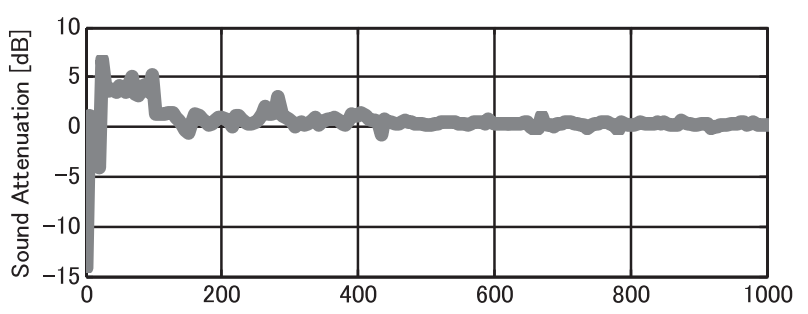

Fig. 17 Experimental results by $\mathrm{H}$-infinity control

Next, $W_{2}$ is selected to attenuate sound between $30 \mathrm{~Hz}$ and $80 \mathrm{~Hz}$ as shown in Fig. 16. Taking care is that inequality (8) and (10) conditions exist. The controller $\mathrm{C}_{2}$ is found above those conditions using MATLAB.

Shown in Fig. 16 are bode diagrams of the closedloop transfer functions $S$ and $T$. Notice that $S$ and $T$ become as specified mostly. Then the simulation using the controller $\mathrm{C}_{2}$ were conducted in $10 \mathrm{kHz}$ of sampling frequency. The simulation result shown in Fig. 17 has the sound attenuating performance of about $4 \mathrm{~dB}$ of maximum in frequency band below $100 \mathrm{~Hz}$.

\section{Conclusions}

An active sound insulation system which has distributed and individually controlled Active Sound Insulation Cells (ASIC) was studied. The secondary source in ASIC was constructed by card-type speakers for weight consumption. Obtained results are as follows:

(1) Uniform sound field in the cell are roughly obtained below the frequency whose wavelength is 4 times of the cell dimension when primary source is operating.

(2) It was proved that the transmitted sound was attenuated at the above frequency region if uniform sound field was constructed by the secondary source.

( 3 ) Type-B has better sound attenuating performance than Type-A. However, some better arrangement of the secondary source needs to be developed.

( 4 ) The ANC experiment result by Filtered-x-LMS algorithm shows that about $10 \mathrm{~dB}$ of the sound attenuation performing was obtained below $400 \mathrm{~Hz}$.

( 5 ) The feedback controller for ANC was systematically designed by $\mathrm{H}$-infinity control theory, and the stable sound attenuating performance of about $4 \mathrm{~dB}$ of maximum was obtained below $100 \mathrm{~Hz}$ by the simulation. 
The future works are creation of a more uniform sound field, improvement of the design technique of the weighting functions for enlarging sound attenuating performance more, and development of the hardware in which sound attenuation can be realized in a larger frequency band.

\section{References}

( 1 ) Nishimura, M., Ohnishi, K., Patrick, W.P. and Zander, A.C., Development of Active Acoustic Treatment (Phase1: Basic Concept and Development of AATCell), Proc. of ACTIVE97, (1997), pp.319-330.

( 2 ) Patrick, W.P., Zander, A.C., Mehta, P.G., Nishimura, M. and Ohnishi, K., Development of Active Acoustic Treatment (Phase2: Duct Test of AAT-Sheet), Proc. of ACTIVE97, (1997), pp.331-344.

( 3 ) Ohnishi, H., Uesaka, K., Ohnishi, K., Teranishi, S. and Nishimura, M., Development of the Noise Barrier with Active Controlled Acoustical Soft Edge — Field Test Using a Loud Speaker and a High Speed Running Truck —, Proc. of Internoise-2000, (2000).

( 4 ) Paurobally, R., Pan, J. and Bao, C., Feedback Control of Noise Transmission through a Double-Panel Partition, Proc. of ACTIVE99, (1999), pp.375-386.

( 5 ) Bao, C. and Pan, J., Mechanisms of Active Attenuation of Noise Transmission through Double Walls, Proc. of ACTIVE97, (1997), pp.965-978.

( 6 ) Jakob, A. and Moser, M., Active Control of DoubleGlazed Windows Part 1: Feedforward Control, Applied Acoustics 64, (2003), pp.163-182.

( 7 ) Jakob, A. and Moser, M., Active Control of DoubleGlazed Windows Part 2: Feedback Control, Applied Acoustics 64, (2003), pp.183-196.

( 8 ) Mizuno, K., Kakuhari, I. and Terai, K., Control Modules Using Piezo Ceramic Loudspeakers to Reduce the Sound Transmission through Walls, The Proc. of ASJ, Fall '03, (2003), pp.591-592. 